\title{
Problematic Diagnosis of a Patient with Tuberculosis Peritonitis
}

\author{
Elieza L. Pramugaria, Iswan Abbas Nusi, Poernomo Boedi Setiawan, Herry Purbayu, Titong \\ Sugihartono, Ummi Maimunah, Ulfa Kholili, Budi Widodo, Husin Thamrin, Amie Vidyani and \\ Muhammad Miftahussurur \\ ${ }^{1}$ Department of Internal Disease, Faculty of Medicine, Universitas Airlangga, Dr. Soetomo Teaching Hospital, Jl. Prof dr. \\ Moestopo 47 Surabaya 60132 Indonesia \\ apji@fk.unair.ac.id
}

Keywords: Tuberculous peritonitis, parietal peritoneum, abdomen, diagnostic problems, FDC therapy

\begin{abstract}
Tuberculosis Peritonitis is a parietal or visceral peritoneal inflammation caused by Mycobacterium tuberculosis bacteria. Pathogenesis Tuberculosis peritonitis is preceded by infection with M. tuberculosis followed by spreading to the peritoneum. This is a report of a young female patient with a major complaint of overall abdominal pain and weight loss accompanied by other non-specific clinical symptoms such as fever, enlargement (ascites), with malnutrition, and a history of the patient's child being treated for tuberculosis lymphadenitis. The investigation showed anemia, erythrocyte sedimentation rate (ESR) and increased CRP, and ascites fluid analysis showed an exudate, ascites glucose ratio with blood $<0.96$. Radiological examination of abdominal ultrasound found ascites, with abdominal CT scan examination finding thickening of the intestinal wall, supporting tuberculosis (TB) with bilateral ovarian cyst appearance. Diagnosis of tuberculosis peritonitis is based on histologic tissue examination by laparotomy. This laparotomy examination is performed because anamnesis, physical examination, and other support are not able to determine a definitive diagnosis. Furthermore, patients receive Fixed Drug Combination (FDC) therapy consisting of Rifampicin, Isoniazid, Pyrazinamide, and Ethambutol for 2 months, followed by a combination of Rifampicin and Isoniazid for 7-10 months. The prognosis of tuberculosis peritonitis is sufficient if the diagnosis is immediate and patients are adequately treated.
\end{abstract}

\section{INTRODUCTION}

Tuberculosis Peritonitis is an inflammation of parietal or visceral peritoneum caused by Mycobacterium tuberculosis and also frequently affects the entire peritoneum, the gastrointestinal system equipment, the mesentery, and the external genital organ. This disease rarely stands alone, but is usually a continuation of the tuberculosis process elsewhere, especially pulmonary tuberculosis (Sharma, 2004.)

The incidence of extrapulmonary $\mathrm{TB}$ is increasing. This is related to the increasing number of people with HIV, population migration, primary resistance to first-line therapy, and also increased use of corticosteroids. One of the most frequent manifestations of extrapulmonary TB is abdominal (bowel, peritoneal, liver, or concomitant) TB, where TB peritonitis is a part of abdominal TB. In the United States, peritoneal involvement is ranked sixth regarding extrapulmonary TB cases. The prevalence of TB peritonitis alone accounts for about 3.5\% of all tuberculosis cases and $31-58 \%$ of cases of abdominal TB. Cases of TB peritonitis are often found in individuals under 40 years old, especially women less than 40 years, with a ratio of women and men is 1.5: 1 (Sharma, 2004., Cavalli, 2016). Complaints suffered by patients with tuberculosis peritonitis may resemble other diseases such as cirrhosis of the liver and carcinoma with less prominent ascites symptoms. The course of the disease progresses slowly with varied and nonspecific clinical manifestations, thus becoming a separate diagnostic problem that results in the disease often becoming undiagnosed or delayed diagnosis with increased morbidity and mortality rates (Onofriescu and Luca, 2014; Srivastava and Almusa, 2014).

The following will present a problematic diagnostic case of a young female patient with tuberculosis peritonitis. 


\section{CASE}

A woman, Mrs. O (33 years old), Javanese, Muslim, working as a health analyst, domiciled in Surabaya was a referral patient from a specialist in internal medicine with an abdominal colic observation, nausea, and vomiting. She was hospitalized on June 9, 2016 in Graha Amerta, Dr. Soetomo General Hospital Surabaya.

Anamnesis: The main complaint in this patient was abdominal pain. Abdominal pain in all abdominal quadrants occurred from 1 day before being hospitalized. Abdominal pain was felt abruptly, sometimes disappearing with complaints of nauseavomiting in the form of water mixed with food. The patient felt that her stomach was slightly enlarged in one month, but she did not feel any complaints because it merely looked like a stomach glut. Her appetite decreased during the illness, but was normal beforehand. The patient also had a fever for one month before being hospitalized.

There were no complaints of coughing, spasms, and night sweats. There was a history of $7 \mathrm{~kg}$ weight loss from 42 to $35 \mathrm{~kg}$ in about 6 months. The patient's urine was normal with a yellowish color, like tea. Defecation was smooth each day with a brownish-yellow color and quite a soft consistency.

The patient had regular periods every month, and did not experience bleeding through the birth canal outside the menstruation period. The first day of the last menstruation was two weeks before admission. The patient had a history of caesarian section surgery when giving birth to her first son 18 months before. Before the patient's illness she never took medications such as drugs for lungs, herbs, narcotics, or other.

The patient's child had a lump in the neck and was on tuberculosis drugs for 5 months because the test result was positive. All family members underwent sputum examination and plain chest photographs to find the source of transmission to the patient's child, but the result was negative.

Physical examination of the patient found a weak general condition, she was compos mentis, GCS 4-5-6, blood pressure 100/60 $\mathrm{mmHg}$ (lying, on right arm), pulse 98, respiration 20x / minute, axillary temperature $380^{\circ} \mathrm{C}, \mathrm{SpO}_{2} 98 \%$. Weight 35 $\mathrm{kg}$, height $160 \mathrm{~cm}$ with IMT 13.67 (underweight). Examination of the head and neck found no anemia, jaundice, cyanosis, or dyspnea. No enlarged lymph nodes and increased jugular venous pressure were found. On examination of the thorax: chest inspection found the form and movement of the chest wall to be symmetrical, no retracted chest; lung: fremitus normal left lung grip, normal percussion, auscultation found vesicular in both lungs field; first and second heart sound is singular no murmurs, gallops, or extra systole. Abdominal inspection: slight distension, darm contour $(+)$, post scar (midline infraumbilical), palpation: defans muscular (-), periumbilical pain $(+)$, undulation $(+)$, chessboard phenomenon (-), percussion: liver $(+)$, shifting dullness $(+)$, auscultation: normal bowel sound $(+)$. On examination of limbs they were warm, dry, red, with no edema or palmar erythema.

Results of laboratory when admitted to emergency room of Dr. Soetomo hospital was hemoglobin 10.0g / dl, MCV 77,1 fL, MCH $23.6 \mathrm{pg}$, leucocytes $6,400 / \mathrm{mm}^{3}$, platelets $333,000 / \mathrm{mm}^{3}$, $84 \%$ granulocytes, lymphocytes $7.1 \%$ (25-40\%), GDA 105g / dl, SGOT 15 U / L, SGPT 12 U / L, direct bilirubin $0.06 \mathrm{mg} / \mathrm{dl}$, total bilirubin $0.18 \mathrm{mg} /$ dl, 3.0g / dL albumin, HBsAg (-), BUN $20 \mathrm{mg} / \mathrm{dl}$, serum creatinine $0.64 \mathrm{mg} / \mathrm{dl}$, sodium $139 \mathrm{mmol} / \mathrm{L}$, potassium $4.5 \mathrm{mmol} / \mathrm{L}$, chloride $109 \mathrm{mmol} / \mathrm{L}$. Urinalysis: SG 1.017, $\mathrm{pH}$ 6, negative glucose, protein (-); negative nitrite; sediments: erythrocytes 0-2 / power field, leucocytes 0-2 / power field, epithelium 1-2 / power field, hyaline -, granular -, bacteria -, yeast -. BGA: $\mathrm{pH}: 7.375 ; \mathrm{pCO}_{2}: 25.5$; $\mathrm{pO}_{2}: 93,5 ; \mathrm{HCO}_{3}: 15, \mathrm{BE}: 2.4 ; \mathrm{SO}_{2}: 97.3$.

Previous laboratory results of June 1, 2016 from private hospitals (1 week before hospitalized with slight fever for 2 weeks): Hemoglobin $10.0 \mathrm{~g} / \mathrm{dl}$, leucocytes 5,250/ mm3, lymphocytes 12.9 , platelets

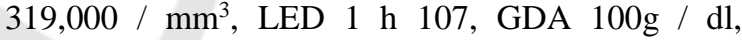
HbA1C 5.8\%, SGOT $30 \mathrm{U} / \mathrm{L}$, SGPT $13 \mathrm{U} / \mathrm{L}$, BUN $20 \mathrm{mg} / \mathrm{dl}$, serum creatinine $0.64 \mathrm{mg} / \mathrm{dl}$, total cholesterol $104 \mathrm{mg} / \mathrm{dL}$, LDL $63 \mathrm{mg} / \mathrm{dL}$, HDL 23 $\mathrm{mg} / \mathrm{dL}$, triglyceride $76 \mathrm{mg} / \mathrm{dL}$, uric acid $2.6 \mathrm{mg} /$ dL hs-CRP $24.8 \mathrm{mg} / \mathrm{dL}$ (hs-CRP $\geq 10$ : possible infection / active inflammation), Anti TB Rapid: negative, Widal: Salmonella Typhi O (+) 1/80, Salmonella Typhi H (-), Salmonella Paratyphi A (-), and Salmonella Paratyphi B (-). Urinalysis: SG $1.010, \mathrm{pH} 6$, glucose (-), protein $(-)$; nitrite $(-)$, ketone (-), urobilinogen (-), bilirubin (-), blood (-), leukocytes (-); sediment: erythrocytes 0 / lp, leukocytes 0 / lp, epithelium 1-2 / lp, hyaline-, granular -, bacteria 1 (0-93). Anti TB Rapid (-). Electrocardiography: Rhythm of 98 x / min, normal axis. Photo thorax: Within normal limits. BO: There was prominent material fecal in the right abdominal cavity and pelvic cavity, with no opaque stone appearing along the urinary tract. Abdominal 
Ultrasound: Ascites no sign of appendicitis, so the presence of retrocaecal appendicitis cannot be excluded. Right / left kidney / Hepar / GB / Lien / pancreas / buli / invisible abnormalities.

Consultation to the surgical section concluded the patient had suspected adhesion intestine postoperative SC, typhoid fever, and malnutrition. The suggestion was to check the abdominal CT scan in GRIU, infusion of RL $1500 \mathrm{cc} / 24 \mathrm{~h}$, injection of metamizole $3 \times 1 \mathrm{~g}$ iv, while fasting, and joint treatment.

Diagnosis: Observation of abdominal colic and observation of ascites suspected adhesion intestine post SC also malnutrition.

Examination Plan: CRP, procalcitonin, albumin, globulin, GDA, HBsAg, anti HCV, FL, sputum BTA and gram. Conducted puncture of ascitic fluid, obtained $25 \mathrm{cc}$ of fluid with reddish color, and planned examination of culture, analysis, and cytology of ascitic fluid.

Therapies given: TKTP1,900 kcal / hr Diet, Amino fluid Infusion 500cc / 24h, Ondansetron injection $1 \mathrm{x}$ 1 ampoule iv, Metamizole injection 1 ampoule iv if pain occurs, Levofloxacin Injection 1x500mg / day iv, Drip multivitamin 1x1 amp / day.

Day 5 of Treatment: Complaints of fever and abdominal pain were absent. Appetite improved. TD 100/60 mmHg, pulse 80x / min, RR 18x / minute, temperature $36.8^{\circ} \mathrm{C}$. Laboratory: albumin $3.0 \mathrm{~g} / \mathrm{dL}$; globulin $3.5 \mathrm{~g} / \mathrm{dL}$, GDA 102, nonreactive HBsAg, non-reactive $\mathrm{HCV}, \mathrm{CRP} 35$, procalcitonin 0.09. Complete feces: soft brown, blood (-), mucus (-), microscopic: erythrocytes (-), leukocytes (-), worms egg (-), amoeba (-), cysts (-), flagellates (-), other (-). Sputum smear negative examination did not find the formation of acid-resistant stem bacteria, sputum gram examination did not find the formation of coccus gram negative or positive. Ascites fluid analysis: ascitic fluid glucose $100 \mathrm{mg} / \mathrm{dL}$, cell count 1,600 cells / uL, mononuclear $61 \%$, 39\% polynuclear, total protein $5.5 \mathrm{~g} / \mathrm{dL}, \mathrm{LDH} 594, \mathrm{pH}$ 8.0; no TB or coccus germs were found. The results of ascitic cytology of ascites obtained the results: the smear contained active mesothelial cells with a round to oval nucleus. There were also seen mononuclear inflammatory cells. The conclusion of ascitic fluid cytology was no malignant cells.

Diagnosis: Observation of colon abdomen and observation of ascites suspected adhesion suspension post SC or tuberculosis peritonitis also malnutrition. The patient underwent a CT scan of the abdomen with contrast and laboratory tests of the latest BUN and serum creatinine. Additional therapy of
Ceftriaxone $2 \mathrm{x} 1 \mathrm{~g}$ iv injection (combination with Levofloxacin) and other therapy were continued.

Day 6 of Treatment: There was no complaint. TD 100/70 $\mathrm{mmHg}$, pulse $80 \mathrm{x} / \mathrm{min}$, RR $20 \mathrm{x} / \mathrm{min}$, tax $36.5{ }^{\circ} \mathrm{C}$. Laboratory: BUN $7 \mathrm{mg} / \mathrm{dL}$, serum creatinine $0.7 \mathrm{mg} / \mathrm{dL}$. Abdominal CT scan results: 1. Diffuse thickening of the bowel wall with attachment and multiloculated ascites. Suggestive peritonitis TB, 2. Multiple paraaortic nodes largest diameter $2.69,3$. Adnexa bilateral left cyst $2.86 \mathrm{x}$ $3.88 \times 4.35 \mathrm{~cm}$ and right $2.86 \times 2 \times 2 \mathrm{~cm}, 4$. Subcentimeter liver cyst. Consultation with the obgyn department led to the plan for laparotomy.

Diagnosis: suspected TB Peritonitis with a bilateral Adnexa cyst (suspicious malignancy) + malnutrition. The patient underwent a DL examination, serum electrolytes, hemostasis phases (as a surgical preparation), and PCR TB examination. Therapy added anti-TB drug (ATD): Rifampicin 1 x 450mg, INH 1 x $400 \mathrm{mg}$, Ethambutol 3 x $250 \mathrm{mg}$, Pyrazinamide 2 x $500 \mathrm{mg}$. However, the patient's family decided to delay taking ATD to await the results of PCR TB first.

Day 7 of Treatment: There was no complaint. TD 99/60 mmHg, pulse 76x / min, RR 20x / min, temperature $36.5{ }^{\circ} \mathrm{C}$. Antenatal cone. Laboratory Hb9.6 g / dL, hematocrit 31.3\%, leucocytes 8,130 / $\mathrm{mm}^{3}$; platelets: $463,000 / \mathrm{mm}^{3}$, neutrophils: $72.4 \%$, $\mathrm{Na} 141 \mathrm{mmol} / \mathrm{L}, \mathrm{K} 4.8 \mathrm{mmol} / \mathrm{L}$, PPT 14.4 (control 14.8), APTT 30.6 (control 28.3).

Consultation in the department of anesthesiology - reanimation: pre-operation was performed with preparation of informed consent of anesthesia and installation of CVC, fasting at 24.00, amino fluid infusion $100 \mathrm{cc} / \mathrm{h}$ since fasting, omeprazole injection $40 \mathrm{mg}$ iv 1 hour before surgery, PRC 2 bag preparation, temperature, tension, pulse, and morning GDA, and praying.

Diagnosis: suspected TB Peritonitis with bilateral Adnexa cyst (suspicious malignancy) + malnutrition + anemia. Additional therapy: transfusion of PRC 1 bag before surgery (due to Hb9.6 g / dL).

Day 8 of Treatment: There were no complaints; the patient had been fasting since night at $24.00 \mathrm{wib}$ for the preparation of surgery. TD $99 / 60 \mathrm{mmHg}$, pulse 80x / min, RR 20x / minute, temperature 36.5 ${ }^{\circ} \mathrm{C}$. Laboratory: GDA: $75 \mathrm{mg} / \mathrm{dL}$.

The patient underwent laparotomy surgery, and during the operation nodules were found allegedly milier small TB image in the intestine and pelvis, and examined Anatomical Pathology and ascites fluid $25 \mathrm{cc}$ for cytologic examination, and installed a drain. Diagnosis: Peritonitis $\mathrm{TB}+$ malnutrition + post transfusion anemia. 
Additional therapy before surgery: Amino fluid infusion 100cc / $\mathrm{h}$ since fasting, omeprazole injection 1 hour before surgery, and other therapy was continued.

Post-surgery the patient received DL, CRP, albumin / globulin (postoperative). Postoperative therapy: infusion of RD5 1,000cc / 24h, drip paracetamol 4 x $500 \mathrm{mg}$ iv, injection of ropivacaine hydrochloride $0.2 \%$ via epidural $5 \mathrm{cc} /$ hour. Injection therapy of levofloxacin was stopped (7th day), replaced orally with: zaldiar $3 \times 1$ tablet, amoxicillin + clavulanic acid $3 \times 500 \mathrm{mg}$, and fentanyl patch $25 \mathrm{mg}$, and other therapy was continued.

Day 10 of Treatment: There were no complaints, but sometimes there was still pain in the area of surgery, with production of a liquid drain of $100 \mathrm{cc}$ with yellowing. TD $99 / 60 \mathrm{mmHg}$, pulse $70 \mathrm{x} / \mathrm{min}$, RR 20x / minute, temperature $36.5^{\circ} \mathrm{C}$. Performed an epidural aff. Laboratory: Hb $11.7 \mathrm{~g} / \mathrm{dL}$, hematocrit $37.7 \%$, leukocytes $7,940 / \mathrm{mm}^{3}$; platelets: $371,000 /$ $\mathrm{mm}^{3}$, neutrophils: $78.9 \%$, Albumin $3.0 \mathrm{~g} / \mathrm{dL}$, globulin $4.2 \mathrm{~g} / \mathrm{dL}$, CRP 63 (normal <6), negative PCR TB. Ascites fluid cytology results: The smear showed the distribution of lymphocyte inflammatory cells, histiocytes, and some mesothelial cells, but no malignant cells. Conclusion: ascitic fluid cytology showed non-malignant cells. Diagnosis: Postoperative laparotomy + Peritonitis TB + malnutrition + post anemia oral therapy continued, while others awaited results of PA.

Day 13 of Treatment: Pain complaints still existed (minimum). Results of a PA biopsy with peritoneal material: the preparation showed fibrous and fatty connective tissue with epithelioid cells forming tubercles with several Langhans datia cells, but no malignant cells. Conclusion: chronic peritonitis tuberculosa.

Infusion therapy D5: Amino fluid $=1: 1 / 24$ hours, planned to administer ATD (Rifampicin 1x450 mg, INH 1 x $400 \mathrm{mg}$, Ethambutol 3x250 mg, Pyrazinamide $2 \times 500 \mathrm{mg}$ ) with complete laboratory examination: DL, LED, SGOT, SGPT, direct and total bilirubin and albumin before ATD was given.

Day 14 of Treatment: No complaints. Laboratory: Hemoglobin $11 \mathrm{~g} / \mathrm{dl}$, leucocytes 7,310 / $\mathrm{mm}^{3}$, platelets $423,000 / \mathrm{mm}^{3}$, granulocytes $82.7 \%$, BUN $11 \mathrm{mg} / \mathrm{dl}$, serum creatinine $0.4 \mathrm{mg} / \mathrm{dl}$, SGOT 25, SGPT 20, direct bilirubin 0.13; total bilirubin 0.36; albumin 3.3g / dL. Diagnosis: Postoperative laparotomy + Peritonitis TB + malnutrition + post anemia.

Treatment: The patient wanted to take a packed ATD (FDC) and wanted to continue ATD therapy at the public health center where the patient is on duty.
Therapy: FDC 1 x 3 tablets and mobilization, infusion aff.

Day 17 of Treatment: No complaints. Patient's plan was KRS with a cover letter delivered to continue treatment to puskesmas. With late diagnosis: $\mathrm{TB}+$ malnutrition + post anemia peritonitis with a message for LFT checks every 2 weeks during the control.

\section{DISCUSSION}

Complaints of abdominal pain in a patient, especially women can be caused by various diseases based on the symptoms that occur, history of disease, and epidemiological data. In addition to diseases that have classic symptoms such as ovarian tumors, cirrhosis of the liver with ascites and spontaneous bacterial peritonitis, and inflammatory bowel disease (IBD), there are still diseases with nonspecific symptoms such as tuberculosis peritonitis (Onofriescu and Luca, 2014; Bolognesi, 2013; Sabooni, 2015).

In tuberculosis peritonitis, the clinical symptoms are neither specific nor varied. Complaints and symptoms occur slowly for months, so the patient is often unaware of his condition. Complaints range from 2 weeks to 2 years with an average of more than 16 weeks (Onofriescu and Luca, 2014; Srivastava and Almusa, 2014). Complaints are also difficult to distinguish from other intra-abdominal diseases. The most common complaints are abdominal pain (73\%) and abdominal / ascites (93\%) followed by decreased appetite and weight, nausea, vomiting, cough, fever (58\%), diarrhea, constipation and night sweats. This disease rarely stands alone, and is often a continuation of the tuberculosis process elsewhere, particularly pulmonary tuberculosis. But often at the time of diagnosis there is established process of tuberculosis in the lungs but it will not be visible for it has been healed first, while the spread is still going on elsewhere (Onofriescu and Luca, 2014; Srivastava and Almusa, 2014; Rathi and Gambhire, 2016).

In the physical examination of patients with tuberculous peritonitis, the most common symptoms are fever, ascites, abdominal swelling, abdominal pain, pallor and fatigue, rhonchi in the lung (right), pleural effusion, hepatomegaly, splenomegaly, intraabdominal tumor, chessboard phenomenon, lymphadenopathy, and involvement of lung \& pleura (on the basis of thorax photo). The phenomenon of chessboard which is a characteristic of tuberculosis peritonitis is in fact not common. The general 
condition of the patient can still be quite good until the skinny and cachexia (depending on the length of the complaint), in women there is often found tuberculosis peritoneum accompanied by tuberculosis in the ovary or tuba, so that the genitals can have signs of inflammation that are often difficult to distinguish from ovarian cysts (Srivastava and Almusa, 2014; Onofriescu and Luca, 2014).

The patient came to the hospital with a major complaint of abdominal pain accompanied by nausea and vomiting. In addition, she also felt abdominal enlargement. Slight fever had been felt for 3 months along with weight loss. There were no complaints of coughing and night sweats. She gave a history of sc surgery 18 months before. The patient's child was undergoing treatment of glandular tuberculosis, but the other family members underwent smear tests and radiology was negative. There were conditions of malnutrition and ascites. Possible conditions experienced by patients can be caused by peritonitis tuberculosa, malignancy, or adhesion (adhesion) intestinal postoperative (considering the patient had previous SC surgery). Among the three possibilities, tuberculosis peritonitis was the first diagnosis when considering the abdominal pain complaints and decreased BB and ascites is a common complaint in tuberculosis peritonitis, especially considering Indonesia is a TB endemic country. Although the patient had had a sputum smear and radiology examinations with previous negative results, this did not exclude the diagnosis since it may be possible that the primary TB process in the lung had healed. Thus, we needed to confirm the diagnosis of the patient by performing other investigations, considering the symptoms that were not typical and similar to each other.

Laboratory examination of tuberculosis peritonitis patients, mostly would found anemia of chronic disease and increased CRP. Other laboratory abnormalities were mild leukocytosis or leukopenia, thrombocytosis, liver disease disorder, and blood sedimentation rate (ESR) increased with an average value of 67.7 and $23 \%$ of patients with an LED score above 100 , whereas on tuberculin testing the results were often negative (Sabooni, 2015; Rai, 2003).

Examination of ascitic fluid analysis generally shows exudate with protein $>3 \mathrm{~g} / \mathrm{dl}$, cell count above 100-3,000 cells / $\mathrm{ml}$ (dominated by lymphocytes, where lymphocytes > 50\% indicate chronic inflammation), LDH is usually increased (> $200 \mathrm{U} / \mathrm{L})$. Purulent ascitic fluid can be found in the majority of patients (81.9\%), as well as serosanguinous ascites in $23.3 \%$ of patients. The examination of acid-resistant bacilli (BTA) resulted in less than $5 \%$ positive results and with liquid culture found less than $20 \%$ positive results. Ascites fluid culture results can be obtained within 4-8 weeks so it is less reliable in diagnosing tuberculosis peritonitis considering the time required more (Cavalli, 2016; Field, 2004). Comparison of ascitic fluid glucose with blood in peritoneal tuberculosis is $<0.96$ while in ascites with other causes is $>0.96$. Reduced $\mathrm{pH}$ of ascitic fluid and elevated lactate levels can be found in tuberculosis peritonitis and is significantly different from ascitic fluid in sterile liver cirrhosis, but $\mathrm{PH}$ examination and lactate content of ascitic fluid is less specific because it is also present in cases of ascites due to malignancy or spontaneous bacterial peritonitis (Sabooni, 2015; Chong, 2005).

Other helpful, rapid and non-invasive ascites are ADA (adenosine deaminase activity), interferon gamma (IFN-Y), and PCR. PCR examination was more frequent because it was more likely to be performed and more readily available than ADA and IFN- $\gamma$, but the sensitivity rate for diagnostic peritonitis of tuberculosis was only $36.3 \%$, lower than IFN- $\gamma(90.9 \%)$, whereas ADA: $18.8 \%$, with the specificity of each $100 \%$. PCR examination gives positive values in $25 \%$ of patients (Cavalli, 2016; Field, 2004).

Tumor markers still need to be checked if ascites are suspected from tumors (malignancy). A common examination is Ca-125. However, Ca-125 is reported to increase both in benign and malignant circumstances, of which approximately $80 \%$ increase in women with ovarian malignancy, $26 \%$ in the first trimester of pregnancy, menstruation, endometriosis, uterine myoma and salpingitis, as well as other gynecological primary cancers such as endometrium, fallopian tubes, endocervix, pancreas, kidney, colon, and also in non-malignant conditions such as chronic renal failure, autosomal disease, pancreas, liver cirrhosis, peritoneal inflammation such as tuberculosis, pericardium and pleura. However, some reports also found elevated Ca-125 levels in patients with tuberculosis peritonitis and found increased levels of $\mathrm{Ca}-125$ with a mean rate of $370.7 \mathrm{ml} / \mathrm{ml}(66.2-907 \mathrm{u} / \mathrm{ml})$ and when there is an increase in serum Ca-125 accompanied with exudative ascitic fluid, with a cell count $>350 / \mathrm{m}^{3}$, dominant lymphocytes, tuberculosis peritonitis may be considered as a diagnosis (Bolognesi, 2013; Gosein, 2013).

The patient's peripheral blood examination showed anemia with elevated CRP (these two parameters are the most common laboratory results 
in TB peritonitis) and increased ESR, but a tuberculin test was not performed. Analysis of ascitic fluid: serosanguinous fluid with $5.5 \mathrm{~g} / \mathrm{dL}$ protein content, increased cell count $(1,600)$, increase in $\mathrm{LDH}$, ascites blood glucose ratio $<0.96 \mathrm{~g}$ / dL, did not obtain decreased $\mathrm{pH}$ of ascites (pH8), and no coccus or BTA germs. PCR-TB gave negative results. Smear sputum found no gram positive and negative gram coccus and smear. The ascitic fluid cytology obtained contained reactive mesothelial cells with a round to oval nucleus. Also seen are mono nuclear inflammatory cells and no malignant cells were found. Overview of laboratory results of both complete blood tests, LED, CRP, and ascites fluid analyses support a tuberculosis peritonitis and cytologic fluid supported ascites with no malignant cells and as per a peritonitis description of tuberculosis. However, these laboratory results are not a definite diagnostic of a tuberculosis peritonitis, so we need to supplement it with other investigations such as radiological examination.

Radiological investigations include chest and abdominal x-ray, abdominal ultrasound, and CT scan. Thorax photo examination is less reliable in the diagnosis of tuberculosis peritonitis, since only $20 \%$ of patients get an overview of active tuberculosis (Onofriescu and Luca, 2014; Onofriescu, 2014). Examination of penetrating rays on the digestive system may be helpful if there are acquired small bowel or bowel abnormalities (Srivastava and Almusa, 2014).

Ultrasonographic (ultrasound) examination can be seen in the presence of free fluid in the peritoneal cavity or localized / fixed (in the form of pockets) in the abdominal cavity, the mass in the ileocecal region and enlargement of the peritoneal retro lymph nodes, abscesses in the abdominal cavity, mesenteric thickening, and omental thickening (Onofriescu and Luca, 2014).

This picture is not specific to tuberculosis peritonitis alone, but if found, it is justifiable to administer ATD therapy empirically. In addition, ultrasound can be used as a closed biopsy tool in diagnosing tuberculosis peritonitis (Field, 2004).

Examination of CT Scan for peritoneal tuberculosis generally finds a sandy peritoneal image and for proof needs to be found together with the clinical symptoms of peritoneal tuberculosis. The presence of a slippery peritoneum with a smooth / minimal thickening accompanied by a grimy omental image indicates a tuberculosis peritonitis, whereas nodular peritoneal thickening (embedded and irregular nodules) suggests a peritoneal carcinoma (Onofriescu and Luca, 2014; Field, 2004).

The results of thorax photo was normal. Abdominal ultrasound results showed ascites, with other organs within normal limits. Abdominal CT scan results: 1. diffuse thickening bowel wall with attachment and multiloculated ascites. Suggestive of peritonitis TB, 2. multiple paraaortic nodes with the largest diameter of $2.69,3$. bilateral adnexa cyst left size $2.86 \times 3.88 \times 4.35 \mathrm{~cm}$ and right $2.86 \times 2 \times 2 \mathrm{~cm}$, 4. subcentimeter liver cyst. This radiological investigation further strengthens the alleged diagnosis of tuberculosis peritonitis. Nevertheless, all these results still cannot confirm the diagnosis of tuberculosis peritonitis itself considering some negative results (such as smear negative sputum, ascites fluid analysis, no TB germ, no thoracic image, and negative PCR) and still the possibility of gynecological malignancy (especially of CT scans suggests bilateral adnexa cysts), so another investigation is needed to confirm the diagnosis of tuberculosis peritonitis or malignancy.

Other investigations that can be performed in determining the exact diagnosis of tuberculosis peritonitis is by peritoneoscopy (laparoscopy). Peritoneoscopy (laparoscopy) is a relatively safe, easy, and best way to diagnose tuberculosis peritonitis especially when the ascitic fluid is obtained and is useful for diagnosing young patients with symptoms of uncertainty. This method is able to diagnose peritonial tuberculosis $85-95 \%$. With directional biopsy, histologic examination can be found and granuloma is $85 \%$ to $90 \%$ of all cases with a sensitivity of close to $100 \%$, and if we perform culture the bacil staining germs will be found almost $75 \%$. More important histologic results are obtained when granuloma is more specific than if found granulomas with caseosa (Cavalli, 2016.).

Description of what can be seen in tuberculosis peritonitis: 1. Small or large tubercles of varying size are widespread on peritoneal and intestinal walls, and can also be found on the surface of the liver or other organs; tubercles may join and are nodules. 2. Adhesions vary from simple to extensive among organs within the peritoneal cavity. It often changes the location of a normal anatomy. Adhesion between the intestines, mesentery, and peritoneum can be very extensive. 3. The surface of the peritoneum often changes to very rough, and sometimes the picture resembles a nodule. 4. Ascites fluid is generally clear yellow, sometimes not clear yet but becomes cloudy, or hemorrhagic (Field, 2004). 
The biopsy is directed to the tubercles in a directional way or on other tissues suspected of having an abnormality by using a special biopsy device and the liquid may be removed. Although in general the peritoneoscopic picture of tuberculosis peritonitis can be determined easily, the picture may resemble other diseases such as carcinomatous peritonitis, so a biopsy should always be attempted and treatment should be given if anatomical pathology results underpin a tuberculosis peritonitis. Peritonoscopy is not always easy to do. The presence of extensive adhesive tissue and the narrowness of the abdominal cavity also complicates the examination making it difficult to recognize the normal anatomical features of the tools and in such circumstances a diagnostic laparotomy should be performed (Onofriescu and Luca, 2014; Rai, 2003).

In the past, exploratory laparotomy was a frequent diagnostic procedure, but nowadays many authors consider surgery only if a simpler procedure does not provide diagnostic certainty or if there are urgent indications such as intestinal obstruction, perforation, presence of purulent ascites (Rai, 2003).

The patients did not undergo peritonoscopy (laparoscopy), but laparotomy and biopsy were performed according to the obstetrics-gynecologist's advice. Observations during laparotomy have been shown to describe nodules suspected of miliary TB images of the small intestine and pelvis, and ascites.

Histopathological examination results obtained fibrous and fatty connective tissue with epithelioid cells forming tubercles with several Langhans datia cells, but no malignant cells. Conclusion: chronic peritonitis tuberculosa. The patient was diagnosed with tuberculosis peritonitis.

Pathogenesis Tuberculosis peritonitis is initiated by $M$. tuberculosis infection followed by spreading to the peritoneum in several ways: 1 . Hematogenous spread (mainly from the lungs), 2. Direct propagation of mesenteric lymph nodes (lymphogenic) or from glandular tube rupture, 3. Direct propagation through infected intestinal wall (germs derived from sputum swallowed by smear positive TB patients), 4. Through the fallopian tubes or infected ovaries. Generally tuberculoid peritonitis occurs through the spread of primary process hematogen (latent infection reactivation "Dorman infection"), not due to direct spreading (Cavalli, 2016; Rathi and Gambhire, 2016).

Based on the pathology there are three forms of tuberculosis peritonitis: exudative, adhesive, and mixed forms: 1. Exudative shape / wet or ascites form a lot, with the main symptoms of enlarged and filled abdominal fluid (ascites), but adhesions are rarely encountered. This form is most common (95.5\%). Tubercles are commonly found, small white-yellowish-colored white, appearing in the peritoneum or in body organ located in the peritoneal cavity. Exudates can form quite a lot, covering tubercle and peritoneum and change the abdominal wall which becomes tense. Ascitic fluid sometimes mixes with blood and looks reddish so there is the possibility of malignancy. Omentum can be affected so that there is thickening and it is palpable like a tumor lump. 2. Adhesive shape / dry or plastic form, less fluid is formed, tubercles are usually larger, and more adhesions occur. The extensive adhesions between the gut and the peritoneum often give a tumor-like appearance, sometimes fistula formation, due to adhesions of the intestinal wall and parietal peritoneum and then necrotic processes that often cause obstruction of the ileus state. 3 . The form of mixture / cyst formed by exudation along with adhesion form a liquid in the pouches of adhesion (Srivastava and Almusa, 2014).

The patient has minimal ascites and from abdominal ct scan were obtained thickening desciption of the intestinal wall with attachment and multiloculated ascites, also the presence of bilateral ovarian cyst which is a picture of pocketed ascites hence she was classified as peritonitis tuberculosa mixture form.

Treatment of tuberculosis peritonitis is similar to pulmonary tuberculosis, i.e. at minimum the patient receives therapy for 6 months. Patients who have not received treatment and are non-resistant to oral antituberculosis (ATD) are treated with first-line regimens consisting of the initial phase including Isoniazid $5 \mathrm{mg} / \mathrm{kgBW} / \mathrm{hr}, 15 \mathrm{mg} / \mathrm{kgBB} / \mathrm{hr}$ Ethambutol, Rifampicin $10 \mathrm{mg} / \mathrm{kgBW} / \mathrm{hr}$, and 25 $\mathrm{mg}$ of Pyrazinamide / $\mathrm{kgBW} / \mathrm{hr}$ given daily for 2 months, then the follow-up phase includes combination of Isoniazid (10 mg / $\mathrm{kgBB} / \mathrm{hr}$ ) and Rifampicin (30mg / kgBB / hr) 3x a week for 4 months. This therapy guide gives good results after 2 months. Treatment of tuberculosis peritonitis can be given 9-12 months (2HRZE / 710RH). The treatment guidelines may use a Fixed Drug Combination (FDC) combination, consisting of a combination of 2 or 4 types of medication in one tablet with a dose according to the patient's weight packed in a package for one patient consisting of an intensive phase every day for 56 days RHZE (150/75/400/275), and advanced stages for 7 to 10 months of RH (150/150) 3x a week. This guide can improve patient compliance (Onofriescu and Luca, 2014; Indonesia, 2014). 
The prognosis of tuberculosis peritonitis is good enough if the diagnosis is immediate and patients are adequately treated (Sharma, 2004; Onofriescu and Luca, 2014).

\section{REFERENCES}

BOLOGNESI, M. A. B., D.. 2013. . Complicated and Delayed Diagnosis of Tuberculous Peritonitis . AMJ CaseRep. , 14:, 109-112

CAVALLI, Z., ET ALL. 2016. Clinical Presentation, Diagnosis, and Bacterial Epidemiology ,of Peritoneal Tuberculosis in two University Hospitalin France. . Brief Report. Infect Dis Ther

CHONG, V. H. A. R., N. 2005. . Tuberculous Peritonitisin Negara Brunei Darussalam. . Original Article. Annals Academy of Medicine Singapore; , 34, p548-52

FIELD, S. A. L., S.. 2004. . Intestinal and Peritoneal Tuberculosis., Lippincot, Williams and Wilkins., Philadelphia:.

GOSEIN, M. A. A. N., D. 2013. Peritoneal Tuberculosis Mimicking Advanced Ovarian Carcinoma: an Important Differential Diagnosisto Consider. BMC Research Notes 6:88. .

INDONESIA., K. K. R. 2014. . Pedoman Nasional Pengendalian Tuberculosis., Jakarta: Kementrian Kesehatan RI

ONOFRIESCU, M. \& LUCA.A., E. A. 2014. . Peritoneal Tuberculosis Difficulties on The Differential Diagnosis Case Report. . European Scientific Jurnal. , , Edittion vol. 10.

RAI, S. A. T., W,M. 2003. . Diagnosis of Abdominal Tuberculosis: The Importance of Laparascopy. Royal Society of Medicine Press: Journal of The Royal Society of Medicine; , 96, 586-588

RATHI, P. A. \& GAMBHIRE, P. 2016. . Abdominal Tuberculosis. . Journal of the Association of Physicians of India., Volume 64

SABOONI, K., ET ALL. 2015. . Tuberculosis Peritonitis with Features of Acute Abdomen in HIV Infection. . International Journal of Mycobacteriology. , 4: , 151153

SHARMA, M. P. A. B., V.. 2004. Abdominal Tuberculosis. . Indian J Med Res. , 120:, 305-315

SRIVASTAVA, U. \& ALMUSA, O., ET ALL. 2014. . Tuberculous Peritonitis. Radiology Case Reports,, 9, 971 